\title{
Strahlen nach Tschernobyl
}

Während des Kalten Krieges fand nur ein atomarer Ernstfall statt, bei dem die schweizerische Alarmorganisation getestet wurde. Am 26. April 1986 ereignete sich in der Nähe der sowjetischen Stadt Tschernobyl (auf dem Gebiet der heutigen Ukraine) in einem Atomkraftwerk ein schwerer Unfall, bei dem es zu einer Reaktorexplosion kam. Vier Tage nach dem Reaktorunfall war die dabei freigesetzte Radioaktivität auch in der Schweiz feststellbar, wobei die gemessenen Werte doppelt und in bestimmten Gegenden sogar vierfach so hoch waren wie normal. ${ }^{1}$ Nicht zuletzt aufgrund dieser auch in der Schweiz feststellbaren Auswirkungen wurde die Atomkatastrophe von Tschernobyl in den Medien, der Politik und der Bevölkerung stark rezipiert. ${ }^{2}$

Beim Reaktorunfall von Tschernobyl handelte es sich um einen katastrophalen atomaren Ernstfall, der zwar geografisch relativ weit von der Schweiz entfernt stattfand, für die zuständigen Behörden des Bundes jedoch auch hier zu einem Problemfall wurde. Weil Tschernobyl den gouvernementalen Umgang mit Strahlen sowohl im Normalfall als auch im Notfall veränderte, laufen im Folgenden zentrale Stränge der vorangehenden Kapitel zusammen. Im Zentrum steht dabei die Frage, welche Folgen Tschernobyl für das Regieren von Strahlen in der Schweiz am Ende des Kalten Krieges zeitigte.

\subsection{Tschernobyl als schweizerischer Problemfall}

Die Weltöffentlichkeit erfuhr erst mit einer zweitägigen Verspätung von der Reaktorexplosion in Tschernobyl. Noch am selben Tag, am 28. April 1986, wurde die Nationale Alarmzentrale in Zürich alarmiert und in der Folge die unter fachlicher Führung der Eidgenössischen Kommission für AC-Schutz (KAC) stehende Alarmorganisation aufgeboten. ${ }^{3}$ Am 30. April löste das Netz für Automatische Dosis-Alarmierung und -Messung, kurz NADAM, aufgrund

1 Vgl. Huber/Jeschki/Prêtre/Völkle 1995, S. 48.

2 Zu den Auswirkungen von Tschernobyl auf Westeuropa: Kalmbach 2014; Kalmbach 2011; Sabrow 2011; Arndt 2010. Für Osteuropa: Arndt 2016. Zu Folgen von Tschernobyl in der Schweiz: Stricker 2012; Moser 2003.

3 Zur Alarmorganisation, der Nationalen Alarmzentrale und der KAC vgl. Kapitel 6.2. 
erhöhter Radioaktivitätswerte Strahlenalarm aus. Der von der Reaktorexplosion in Tschernobyl verursachte radioaktive Niederschlag hatte auch die Schweiz erreicht. Aufgrund einer wahrgenommenen starken Beunruhigung und Verunsicherung in der Bevölkerung richteten die Bundesbehörden am 6. Mai schließlich verschiedene Sorgentelefone ein. Zudem beauftragte der Bundesrat die Generaldirektion der Schweizerischen Rundspruchgesellschaft, Sendungen zu produzieren, in denen im Radio und Fernsehen live Fragen von Hörerinnen und Hörern bzw. Zuschauerinnen und Zuschauern beantwortet werden sollten. ${ }^{4}$

Die Messorganisation der Alarmorganisation, das heißt der Einsatz der Frühwarnposten, der bereits bestehenden NADAM-Stationen und der mobilen Messwagen, aber auch der Labororganisation und der aufgebotenen militärischen Mittel, mit denen die Radioaktivität in der ganzen Schweiz kontinuierlich gemessen und daraus fortlaufend die Verstrahlungslage bestimmt wurde, bewährte sich. ${ }^{5}$ So gab die KAC bereits seit dem 29. April täglich ein Bulletin über die Strahlensituation in der Schweiz heraus. Trotzdem wurde die Alarmorganisation seitens der Medien und der Politik teilweise scharf kritisiert. ${ }^{6}$ Diese Kritik bezog sich indessen weniger auf deren fachliche Arbeit, als vielmehr auf organisatorische Schwachstellen, welche insbesondere bei der Informationstätigkeit deutlich zutage traten. ${ }^{7}$ Dies zog eine Umdeutung des Ereignisses von Tschernobyl nach sich, indem schließlich nicht mehr die lückenhafte sowjetische Informationspolitik, sondern diejenige der schweizerischen Regierung und Behörden als ungenügend taxiert wurde. Der Historiker Marius Stricker gelangt deshalb zu dem Schluss, in der Schweiz habe Tschernobyl in erster Linie als „Informationskatastrophe“ eine prägende Wirkung entfaltet. ${ }^{8}$ Dafür erwiesen sich im Wesentlichen zwei Gründe als ausschlaggebend: Erstens war die Informationszentrale der Alarmorganisation schlecht geplant und organisiert und deshalb ständig überlastet, was sich durch personelle Engpässe zusätzlich verschärfte. So war der Informationsfluss nicht nur zwischen den Bundesbehörden, den Kantonen, den Medien und der Bevölkerung gestört, sondern auch innerhalb der verschiedenen Teile der Alarmorganisation selbst. Zweitens waren die Informationen der Bundesbehörden verwirrend und bisweilen gar widersprüchlich. Dies zeigte sich etwa daran, dass die KAC keine einheitlichen Größen und Maßeinheiten

\footnotetext{
4 Vgl. Stricker 2012, S. 26, S. 28 und S. 42.

5 Vgl. CH-BAR\#E33ooC\#1996/294\#799*, Folgerungen aus dem KKW-Unfall von Tschernobyl: Schlussbericht, 1.12.1987. Zur Messorganisation der Alarmorganisation vgl. Kapitel 6.2.

6 Vgl. Stricker 2012, S. 29, S. 33, S. 39 und S. 71.

7 Vgl. CH-BAR\#E3310A\#2OO3/2O9\#48*, Antrag des EDI, 8.4.1987.

8 Vgl. Stricker 2012, S. 28 f. und S. 36.
} 
für Radioaktivitäts- und Grenzwerte verwendete und so insbesondere im Vergleich zur Bundesrepublik Deutschland der Eindruck entstand, in der Schweiz würde die Verstrahlungslage verharmlost. Die mediale Berichterstattung verstärkte diese Wahrnehmung, weshalb die Medien - so Stricker - gewissermaßen als „Koproduzenten der Informationskatastrophe“ fungiert hätten. ${ }^{9}$ Insgesamt resultierte aus diesen Informationsdefiziten ein Vertrauensverlust in den Bundesrat und die Bundesbehörden und ihre öffentlich auftretenden Experten, deren Glaubwürdigkeit angeschlagen schien. ${ }^{10}$ Diese Informationsprobleme, die anlässlich des atomaren Ernstfalls von Tschernobyl zutage traten, sind indessen wenig erstaunlich. Vielmehr lassen sie sich - wie in dieser Arbeit immer wieder deutlich wurde - in eine Reihe von Organisationsmängeln und Koordinationsproblemen im Alarmierungs- und Kommunikationsbereich einordnen, wie sie insbesondere anlässlich verschiedener Landes- und Gesamtverteidigungsübungen feststellbar waren.

Werden der Reaktorunfall von Tschernobyl und die Reaktionen, welche dieses Ereignis in der Schweiz auslöste, in den größeren Kontext der Mitte der 198oer Jahre immer noch laufenden gesellschaftlichen Auseinandersetzungen um den Bau von Kernkraftwerken gestellt, lassen sich folgende Phasen unterscheiden: Bis zum Reaktorunfall von Tschernobyl Ende April 1986 herrschte zwischen den Gegnerinnen und Gegnern und den Befürworterinnen und Befürwortern der Kernenergie eine Pattsituation vor, wobei sich mit der Ablehnung der (zweiten) Atominitiative im September 1984, die gegen den Bau von Atomkraftwerken gerichtet war, die befürwortende Seite tendenziell im Aufwind befand. Zwischen Mai 1986 und März 1988 löste der Unfall von Tschernobyl einen zweiten Höhepunkt der Atomenergiediskussion aus, welcher durch zwei Sondersessionen des Bundesparlamentes, die Lancierung von zwei eidgenössischen Volksinitiativen und eine hohe öffentlichmediale Aufmerksamkeit gekennzeichnet war. Daraufhin beschlossen die dominierenden bürgerlichen Bundesratsparteien im April 1988 einen radikalen Kurswechsel, indem sie nun ebenfalls dafür plädierten, auf den umstrittenen Bau des Kernkraftwerkes Kaiseraugst zu verzichten. Mit der Annahme der sogenannten Moratoriumsinitiative wurde im September 1990 schließlich ein vorübergehender Stopp beim Atomkraftwerkbau beschlossen. ${ }^{.1}$ Insgesamt bewirkte die Reaktorkatastrophe von Tschernobyl - wie der Historiker Patrick Kupper betont - bezüglich der Bewertung der Atomenergie in der Schweiz einen „längerfristig wirksame[n] Einstellungswandel in der Öffentlichkeit“. ${ }^{12}$

\footnotetext{
$9 \quad$ Ebd., S. 54, S. 56-59 und S. 77, Zitat S. 77.

10 Vgl. ebd., S. 73 f.

11 Vgl. Moser 2003, S. 185-187. Gleichzeitig wurde eine Ausstiegsinitiative abgelehnt.

12 Kupper 2oo3a, S. 245.
} 
Doch nicht nur die Öffentlichkeit und die Medien, sondern auch die Politik reagierte umgehend auf den Reaktorunfall von Tschernobyl. Davon zeugen eine Vielzahl von parlamentarischen Vorstößen und die beiden Sondersessionen der eidgenössischen Räte vom Juni und Oktober 1986. In der ersten Debatte nach dem Reaktorunfall wurden in zwanzig dringlichen Interpellationen über zweihundert Fragen gestellt, deren Inhalte vom Ablauf des Ereignisses und den Schutzmaßnahmen des Bundes über die gesetzlichen Grundlagen und die Informationspolitik bis hin zu gesundheitlichen, wirtschaftlichen und energiepolitischen Auswirkungen reichten. Der Bundesrat kündigte in seiner Antwort auf diese Interpellationen ein 12-Punkte-Programm an, „in welchem wesentliche Aspekte der Krise und ihrer Bewältigung auf Bundesebene kritisch untersucht und Verbesserungen realisiert werden sollten. ${ }^{\text {“13 }}$ Dieses Programm sah unter anderem vor, zügig bestimmte Gesetze und Verordnungen zu erarbeiten oder aufgrund der Erfahrungen von Tschernobyl zu revidieren, die KAC personell und materiell zu verstärken, eine Informationszentrale zu schaffen, Notfallkonzepte für die ganze Bevölkerung zu erstellen sowie eine Harmonisierung von internationalen Strahlenschutzregelungen und -konzepten anzustreben. ${ }^{14}$

Ähnlich wie nach dem Kernkraftwerkunfall in Harrisburg befasste sich auch die Geschäftsprüfungskommission des Nationalrates mit der Nuklearkatastrophe von Tschernobyl. Ihre Untersuchung sah vor - wie sie dem Bundesrat im Juni 1986 mitteilte - sich "mit den Folgerungen zu befassen, welche die Bundesverwaltung daraus [aus dem Kernkraftwerkunfall von Tschernobyl] zieht. ${ }^{\text {15 }}$ Der Bundesrat stellte der Geschäftsprüfungskommission im Dezember 1986 sowie im März und im Juli 1987 einen Zwischenbericht und im Dezember 1987 einen Schlussbericht zu, welche über die aus dem Reaktorunfall gezogenen Folgerungen sowie über den Stand der Realisierung des 12-Punkte-Programmes informierten. ${ }^{16}$ Als der Schlussbericht des Bundesrates vorlag, begann eine Arbeitsgruppe der Geschäftsprüfungskommission mit ihren materiellen Prüfungen. An zwei zweitägigen Hearings befragte sie die verantwortlichen Beamten des Bundes sowie einige weitere Experten zu Fragen der Sicherheitsstandards, bezüglich des Systems der Grenzwerte und Maßnahmen, der Qualität der Informationspraxis der Behörden in

\footnotetext{
13 CH-BAR\#E330oC\#1996/294\#799*, Folgerungen aus dem KKW-Unfall von Tschernobyl: Schlussbericht, 1.12.1987.

14 Vgl. ebd., Folgerungen aus dem KKW-Unfall von Tschernobyl (insbesondere 12-PunkteProgramm), [22.12.1986].

15 Ebd., Schreiben der Geschäftsprüfungskommission des Nationalrates an den Bundesrat, 19.6.1986.

16 Vgl. ebd., Folgerungen aus dem KKW-Unfall von Tschernobyl: Schsbericht, 1.12.1987.
} 
Krisenfällen, der Funktionsweise der Alarmorganisation sowie zu den Lehren in Bezug auf die Sicherheit der schweizerischen Kernkraftwerke. Daraus resultierte im November 1988 ein Bericht, in welchem die Geschäftsprüfungskommission des Nationalrates achtzehn Maßnahmen empfahl, welche unter anderem das System der Grenzwerte, die Einsatzorganisation bei erhöhter Radioaktivität, die Information der Öffentlichkeit und die Sicherheit der Kernkraftwerke betrafen. ${ }^{17}$ Dieser Bericht und die Stellungnahme des Bundesrates dazu wurden im Frühjahr 1989 in den eidgenössischen Räten behandelt. ${ }^{18}$ Damit gelangte das Thema Tschernobyl in der öffentlich-politischen Diskussion vorläufig an ein Ende. Was indessen das Regieren von Strahlen betraf, so hatte das Ereignis von Tschernobyl Folgen, welche über das Ende des Kalten Krieges hinausreichten.

\subsection{Regieren von Strahlen im Normalfall und im Notfall nach 1986}

Die Atomkatastrophe von Tschernobyl fungierte als Katalysator für prägende Reorganisationen und Umstrukturierungen auf dem Gebiet des Strahlenschutzes. Diese hatten sich zwar teilweise bereits seit den 1970er Jahren abgezeichnet, nach Tschernobyl wurden sie indessen unverzüglich in Angriff genommen. Vor allem die Dispositive zur Überwachung, zur Regulierung und zur Alarmierung erfuhren im Zuge der schweizerischen Aufarbeitung dieses gravierenden Reaktorunfalls wesentliche Anpassungen und Veränderungen.

\section{Überwachen}

Der Reaktorunfall von Tschernobyl führte zu einer Professionalisierung und Aufwertung des Strahlenschutzes und der Strahlenüberwachung in der Bundesverwaltung. So wurde die Sektion für Strahlenschutz am 1. Januar 1987 zur Abteilung Strahlenschutz des Bundesamtes für Gesundheitswesen (BAG) aufgewertet. ${ }^{19}$ Gleichzeitig wurde ihr die neu geschaffene Sektion Überwachung der Radioaktivität unterstellt. Letztere bestand aus der ehemaligen Laboreinheit der Eidgenössischen Kommission zur Überwachung der Radioaktivität (KUeR) in Fribourg, die nun als Verwaltungssektion in die

17 Vgl. CH-BAR\#E330oC\#1996/294\#801*, Bericht der Geschäftsprüfungskommission des Nationalrates an den Bundesrat (Entwurf), 16.9.1988.

18 Vgl. ebd., Stellungnahme des Bundesrates zum Bericht der Geschäftsprüfungskommission, 27.12.1988.

19 Vgl. Müller 1989, S. 33. 
Abteilung Strahlenschutz integriert wurde. ${ }^{20}$ Im April 1987 wurde zudem eine Änderung von Artikel 45 der Schweizerischen Strahlenschutzverordnung vorgenommen. ${ }^{21}$ Dieser Artikel hatte bisher festgehalten, dass die KUeR für die Radioaktivitätsüberwachung der Umwelt verantwortlich sei. Nun sollte die Verantwortung für die dauernde Überwachung der Radioaktivität der Umwelt beim BAG liegen. ${ }^{22}$

Die nach wie vor bestehende KUeR wollte sich mit diesen Kompetenz- und Ressourcenbeschneidungen nicht einfach abfinden, weshalb es zwischen ihr und dem BAG zu länger andauernden Auseinandersetzungen kam. In einem Schreiben an den Vorsteher des Eidgenössischen Departements des Innern (EDI), Bundesrat Flavio Cotti, bestand die KUeR insbesondere darauf, „ihre bisherige Unabhängigkeit nach aussen glaubhaft bewahren“ zu können. ${ }^{23}$ Dazu wollte sie weiterhin verwaltungsunabhängig agieren können und verlangte deshalb, dass ihr die Sektion Überwachung der Radioaktivität fachlich (wenn auch nicht administrativ) wieder unterstellt werde. ${ }^{24}$ Im Prinzip wollte die KUeR den bisherigen Status quo somit auch - respektive gerade - nach dem Reaktorunfall von Tschernobyl beibehalten. Demgegenüber argumentierte das BAG, das Ereignis von Tschernobyl habe gezeigt, „dass dauernde Vollzugsaufgaben in der Regel von der Verwaltung und nicht von einer Kommission mit nebenamtlich tätigen Mitgliedern wahrgenommen werden sollen. ${ }^{25}$ Die Rolle der KUeR sah das BAG lediglich noch in beratender Funktion.

Auch die Organisation der Überwachung der Radioaktivität der Lebensmittel wurde im Nachgang zum Reaktorunfall von Tschernobyl professionalisiert. Ab dem 1. Juli 1989 übernahm der Verband der Kantonschemiker der Schweiz die Aufgaben der seit 1957 bestehenden, jedoch auf freiwilliger Mitarbeit beruhenden Arbeitsgemeinschaft zur Überwachung der Radioaktivität der Lebensmittel. ${ }^{26}$ Auch für diese neue Zuständigkeit gab die Kernkraftwerk-

20 Vgl. CH-BAR\#E3300C\#2OO2/40\#561*, Schreiben der Mitglieder der KUeR an A. Egli, 11.12.1986. Die Überführung des Labors der KUeR in eine Verwaltungseinheit war im Zusammenhang mit einer beabsichtigten Reorganisation der Sektion für Strahlenschutz bereits seit mehreren Jahren ein Thema gewesen. Vgl. CH-BAR\#E330oC\#2002/40\#550*, Aktennotiz betreffend Schaffung einer Sektion Radioaktivitätsüberwachung, 23.4.1982.

21 Vgl. zu Art. 45 der Schweizerischen Strahlenschutzverordnung auch Kapitel 3.3.

22 Vgl. CH-BAR\#E3300C\#2OO2/4O\#561* Antrag des EDI, 10.10.1989.

23 CH-BAR\#E330oC\#2002/40\#564*, Schreiben von H. Loosli an F. Cotti, 21.2.1989. Vgl. auch ebd., Schreiben von H. Loosli an E. Marthaler, 16.2.1988; CH-BAR\#E33OoC\#2002/40\#561*, Schreiben der Mitglieder der KUeR an A. Egli, 11.12.1986.

24 Vgl. CH-BAR\#E 33 OOC\#2OO2/4O\#564*, Schreiben von H. Loosli an E. Marthaler, 16.2.1988.

25 Ebd., Schreiben von B. Roos an Ed. Marthaler, 30.3.1988.

26 Die Kontrolle der Lebensmittel ist eine Aufgabe der Kantone, nicht des Bundes. Zur Arbeitsgemeinschaft zur Überwachung der Radioaktivität der Lebmittel vgl. Kapitel 3.2. 
katastrophe von Tschernobyl den Ausschlag. So betonte der Chef der Abteilung Lebensmittelkontrolle des BAG, die Erfahrungen von Tschernobyl hätten gezeigt, dass bei erhöhter Radioaktivität eine Übertragung allfällig notwendiger Maßnahmen im Lebensmittelsektor an die kantonalen Laboratorien der Lebensmittelkontrolle „sinnvoll und zweckmässig“ sei. ${ }^{27}$

Am Beispiel der Zurückstufung der KUeR und der Eingliederung ihres ehemaligen Labors in die Verwaltung sowie der Neuorganisation der Lebensmittelüberwachung zeigt sich exemplarisch das Ende der Ära der außerparlamentarischen Expertenkommissionen und anderer Milizorgane. $\mathrm{Zu}$ Beginn des Kalten Krieges hatte die Strategie der Bundesverwaltung mangels eigener Expertise wesentlich darin bestanden, gouvernementale Aufgaben an mit entsprechenden externen Experten besetzte Milizgremien abzugeben. Seit dem Ende der 1970er bzw. dem Anfang der 198oer Jahre gab es indessen politische Bestrebungen, die Anzahl außerparlamentarischer Kommissionen zu reduzieren bzw. deren Tätigkeitsfelder auf beratende Funktionen einzuschränken. ${ }^{28}$ Im letzten Jahrzehnt des Kalten Krieges erlitten die Expertenbzw. Milizkommissionen dann nicht zuletzt aufgrund der steigenden Komplexität der zu bearbeitenden Probleme einen allgemeinen Bedeutungsverlust. ${ }^{29}$ Nun wollten - und konnten - Verwaltungsorgane wie das BAG oder die Kantonschemiker Kernaufgaben wie die Radioaktivitätsüberwachung selbst übernehmen. Der Reaktorunfall von Tschernobyl wirkte hier beschleunigend auf schon bestehende Professionalisierungspläne ein oder stieß derartige Bestrebungen an.

\section{Regulieren}

Als Katalysator fungierte Tschernobyl auch im Bereich der Strahlenregulierung. 1994 trat das neue Strahlenschutzgesetz in Kraft, welches die bereits 1982 beschlossene Trennung der beiden lange Zeit miteinander verknüpften Bereiche

27 CH-BAR\#E3310A\#2003/209\#106*, Scheiben von H. R. Strauss an die Kantonalen Laboratorien der Schweiz, an die Lebensmittelinspektorate der Kantone, an die Lebensmittelkontrolle des Fürstentums Liechtenstein, an die Kantonstierärzte der Schweiz, an die Mitglieder und Experten der KUeR und weitere interessierte Kreise, 4.7.1989. Vgl. auch CH-BAR\#E3310A\#2003/209\#480*, Schreiben von B. Michaud an die kantonalen Laboratorien der Schweiz, an die Lebensmittelinspektorate der Kantone und an die Lebensmittelkontrolle des Fürstentums Liechtenstein, 20.10.1988, und Grundsätze der Zusammenarbeit zwischen der Einsatzorganisation des Bundes und den kantonalen Laboratorien bei erhöhter Radioaktivität im Ereignisfall, ohne Datum.

28 Vgl. bspw. die entsprechenden Dokumente in: Archiv BAG, 18.1.1.37, Änderung der Strahlenschutzverordnung sowie des Reglements der Eidg. Kommission für Strahlenschutz.

29 Vgl. Germann 2002. 
Strahlenschutz und Atomenergienutzung definitiv festschrieb. ${ }^{30}$ Bemerkenswert ist, dass der Vorentwurf für das neue Gesetz, welcher noch vor dem Reaktorunfall in Tschernobyl vorlag, nicht mehr von einer juristischen Expertenkommission oder der Eidgenössischen Kommission für Strahlenschutz (EKS), sondern in erster Linie von Angestellten der Bundesverwaltung erarbeitet wurde. ${ }^{31}$ Dieses Vorgehen stellt ein weiteres Indiz für den bereits erwähnten Trend dar, Tätigkeiten von Milizkommissionen in den 198oer Jahren zunehmend in die Verwaltung zu verlagern.

Der verwaltungsinternen Arbeitsgruppe, welche den Vorentwurf ausarbeitete, gehörten Vertreter des Bundesamtes für Justiz, des Bundesamtes für Energiewirtschaft, der Hauptabteilung für die Sicherheit der Kernanlagen, der Schweizerischen Unfallversicherungsanstalt, des EDI und des BAG sowie der EKS an, wobei der Chef der Sektion für Strahlenschutz Hans-Rudolf Stadelmann den Vorsitz übernahm. ${ }^{32}$ Mit Serge Prêtre von der Hauptabteilung für die Sicherheit der Kernanlagen und dem Präsidenten der EKS Gerhart Wagner hatten mehrere Mitglieder der Arbeitsgruppe schon bei der Strahlenschutzverordnung von 1976 mitgewirkt. ${ }^{33}$ Wagner war sogar bereits an der Verordnung von 1963 und den Technischen Richtlinien von 1954 maßgeblich beteiligt gewesen und kann als die prägende Figur der schweizerischen Strahlenregulierung während des Kalten Krieges bezeichnet werden.

Ende November 1985 ging der Vorentwurf der Arbeitsgruppe in die Vernehmlassung, die bis Ende Juni 1986 dauerte. Der Reaktorunfall von Tschernobyl fiel somit mitten in die Vernehmlassungsfrist. Zwar hielt das EDI in seinem Auswertungsbericht fest, der Vorentwurf sei „als Ganzes positiv aufgenommen" worden. Die nun vollzogene Trennung von Strahlenschutz und Kernenergie sei „von keiner Seite bestritten“ und die Absicht, den Strahlenschutz nun auf Gesetzesebene und nicht wie bisher auf der Stufe der Verordnung zu regeln, werde sogar „[a]usdrücklich begrüsst". Trotzdem hätten einzelne Abschnitte oder Artikel „eine sehr vielfältige und teilweise auch massive Kritik“ erfahren. Hier stehe - wie das EDI weiter ausführte - „bei zahlreichen Vernehmlassern das Katastrophenereignis von Tschernobyl mit seinen auch in der Schweiz festgestellten Folgen im Vordergrund““. ${ }^{34}$

$30 \quad$ Vgl. zu dieser Trennung Kapitel 4.3.

31 Vgl. CH-BAR\#E3310A\#2003/209\#22*, Schreiben von U. Frey an den Chef der Sektion für Strahlenschutz, 14.1.1983, und Protokoll der 1. Sitzung der Arbeitsgruppe für die Ausarbeitung eines Strahlenschutzgesetzes, 28.3.1983.

32 Vgl. ebd., Schreiben von U. Frey an den Chef der Sektion für Strahlenschutz, 14.1.1983.

33 Vgl. ebd., Protokoll der 1. Sitzung der Arbeitsgruppe für die Ausarbeitung eines Strahlenschutzgesetzes, 28.3.1983.

34 CH-BAR\#E3310A\#2003/209\#29*, Auswertung der Vernehmlassung zum Vorentwurf des EDI zu einem Strahlenschutzgesetz, ohne Datum. 
Den eingegangenen Stellungnahmen ließen sich vier Kritikpunkte entnehmen: Erstens müsse die Informationspflicht gegenüber der Bevölkerung und den Kantonen durch die Organe des Bundes gesetzlich verankert werden, zweitens seien die Kompetenzen zwischen Bund und Kantonen klarer abzugrenzen, drittens solle ein differenziertes Maßnahmenkonzept für den Alarmfall ausgearbeitet werden und viertens brauche es eine internationale Vereinheitlichung bestimmter Konzepte, Normen und Regelungen, etwa im Bereich der Haftpflicht. ${ }^{35}$ Die als ungenügend wahrgenommene Information von Öffentlichkeit und Kantonalbehörden sowie die aufgedeckten Mängel in der Alarmorganisation wurden indessen nicht nur in der Vernehmlassung kritisiert. Vielmehr spiegelten die Vernehmlassungsantworten diesbezüglich die prägenden Argumente der medialen und politischen Debatte wider.

Interessant ist nun, dass der Vorentwurf, der wie erwähnt bereits vor dem Ereignis von Tschernobyl formuliert worden war, schon eine stärkere Gewichtung einer möglichen gefährlichen Erhöhung der Radioaktivität enthielt. So sollte das neue Strahlenschutzgesetz wichtige Bestimmungen von Verordnungs- auf Gesetzesstufe heben und Möglichkeiten für Vorschriften und Maßnahmen schaffen, für welche die gesetzlichen Grundlagen bis dahin gefehlt hatten. ${ }^{36}$ Die kritischen Vernehmlassungsrückmeldungen forderten bezüglich der Vorbereitung auf einen atomaren Notfall also nichts genuin Neues; vor dem Hintergrund „der mit dem Reaktorunglück von Tschernobyl gemachten Erfahrungen“ erhielten die vorgeschlagenen Bestimmungen jedoch bedeutend mehr Aufmerksamkeit. ${ }^{37}$ Verschiedene Entwicklungen in der Strahlenschutzgesetzgebung, die schon seit einiger Zeit im Gang gewesen waren, erlangten nach dem Reaktorunfall von Tschernobyl somit eine erhöhte Dringlichkeit.

Die Nuklearkatastrophe von Tschernobyl führte dazu, dass die Arbeit am Strahlenschutzgesetz und die Schaffung einer neuen Strahlenschutzverordnung erste Priorität erhielten. ${ }^{38}$ Das neue Strahlenschutzgesetz trat schließlich 1994 fast gleichzeitig mit der ebenfalls neuen Strahlenschutzverordnung in Kraft. ${ }^{39}$ Im Vernehmlassungsverfahren zu dieser neuen Verordnung bildete die Radioaktivität von Lebensmitteln einen der kontrovers diskutierten Punkte. Wie eine Pressemitteilung des EDI festhielt, waren

35 Vgl. ebd., Antrag des EDI, 15·5.1987.

36 Vgl. CH-BAR\#E3310A\#2003/209\#24*, Antrag des EDI, 18.10.1985.

37 CH-BAR\#E3310A\#2003/209\#30*, Antrag des EDI, 25.1.1988. Vgl. auch CHBAR\#E330oC\#1996/294\#799*, Folgerungen aus dem KKW-Unfall von Tschernobyl: Schlussbericht, 1.12.1987.

38 Vgl. CH-BAR\#E330oC\#1996/294\#799*, Folgerungen aus dem KKW-Unfall von Tschernobyl (insbesondere 12-Punkte-Programm), [22.12.1986].

39 Vgl. CH-BAR\#E3310A\#2003/209\#44*, Beschluss des Bundesrates, 22.6.1994. 
radioaktive Lebensmittel "seit Tschernobyl ein aktuelles Thema“. ${ }^{40}$ Diese Feststellung lässt außer Acht, dass die Frage radioaktiver Lebensmittel schon während der Fallout-Debatte zur Zeit der oberirdischen Atomwaffentests thematisiert worden und damit als Problem bereits seit Jahrzehnten bekannt war. ${ }^{41}$ In der Schweiz gelangte die Regulierung der Radioaktivität in Lebensmitteln jedoch erst durch den Reaktorunfall von Tschernobyl auf die gouvernementale Agenda.

Dementsprechend sah die neue Strahlenschutzverordnung nun erstmals vor, Toleranz- und Grenzwerte für radioaktive Nuklide in Lebensmitteln festzulegen. Umweltverbände hielten die vorgeschlagenen Grenzwerte - so das EDI im Bericht über die Vernehmlassungsergebnisse - für „völlig unannehmbar“, da sie "deutlich höher“ seien als die „,Katastrophen-Toleranzwerte“", die der Bundesrat nach Tschernobyl temporär festgelegt hatte. „Bedenklich“ sei für die Umweltorganisationen - wie das EDI weiter ausführte - zudem die Einführung von Toleranzwerten für Radionuklide in Lebensmitteln, zumal diese Werte nicht als Toleranz-, sondern als Grenzwerte bezeichnet werden müssten. ${ }^{42}$

Während Umweltorganisationen die geplanten Grenz- und Toleranzwerte also aus grundsätzlichen Überlegungen ablehnten, argumentierten Vertreter aus Wirtschaftskreisen in entgegengesetzter Richtung. So verlangte eine Stellungnahme „das vorläufige Zurückstellen“ der Bestimmungen über die Radioaktivität in Lebensmitteln mit der Begründung, dass bestimmte Vorschriften "nicht EG- [Europäische Gemeinschaft-] kompatibel“ seien. Die Strahlenschutzgesetzgebung der Europäischen Gemeinschaft kenne beispielsweise keine Grenzwerte für natürliche Nuklide wie Tritium oder Kohlenstoff-14. Solche Abweichungen würden somit „unweigerlich zu neuen Handelshindernissen führen“ ${ }^{43}$ Eine andere Stellungnahme begrüßte zwar die konsequente Angleichung der Grenzwerte an diejenigen der Europäischen Gemeinschaft, zweifelte jedoch grundsätzlich an deren Berechtigung. Die Werte seien „viel zu tief angesetzt“, wodurch sie „den Kontrollorganen einen viel zu grossen Spielraum zur Anordnung von Auflagen für die Verwertung" geben würden. ${ }^{44}$ Für die Wirtschaftsorganisationen waren also zwei Argumente wesentlich: Die Bestimmungen über die zulässige Radioaktivität in Lebensmitteln durften die

\footnotetext{
40 CH-BAR\#E3310A\#2003/209\# $38^{*}$, Presserohstoff: Strahlenschutzverordnung; Vernehmlassung, 22.12.1992.

41 Vgl. dazu Kapitel 3.2.

42 CH-BAR\#E3310A\#2003/209\# $38^{*}$, Bericht über die Ergebnisse des Vernehmlassungsverfahrens zur Strahlenschutzverordnung, Oktober 1993.

43 Ebd.

44 Ebd.
} 
Handelsfreiheit nicht einschränken, und das schweizerische Recht musste den europäischen Normen entsprechen. Der letzte Punkt entsprach einer grundsätzlichen Forderung, wie das EDI betonte: Seitens der Vernehmlasser werde darauf hingewiesen, „dass die Verordnung eurokompatibel sein müsse. Eine ,Helvetisierung 'von internationalen Normen sei abzulehnen; der übermässige schweizerische Perfektionismus verursache hohe Kosten, ohne dass entsprechende praktische Vorteile daraus entstünden.“45 Diese Argumente der Wirtschaftsvertreter stießen bei den Bundesbehörden auf offene Ohren. So überprüfte das EDI das Verhältnis zum europäischen Recht und kam dabei zu dem Schluss, „[i]m Hinblick auf einen möglichst freien Personen- und Warenverkehr" würden sich "keine Probleme bzw. technischen Handelshemmnisse“ ergeben. ${ }^{46}$

Die Strahlenschutzvorschriften bezüglich der Radioaktivität von Lebensmitteln stehen exemplarisch dafür, dass die Kompatibilität von schweizerischem mit europäischem Recht zu Beginn der 199oer Jahre ein zunehmend virulentes Problem darstellte. ${ }^{47}$ Zwischen den Bundesbehörden und den Wirtschaftsvertretern war diesbezüglich ein Konsens dahingehend vorherrschend, dass eine rechtliche Angleichung sinnvoll und gerade aus ökonomischer Sicht zwingend erforderlich sei. Das Beispiel der schweizerischen Strahlenschutzverordnung von 1994 zeigt somit erneut, dass die voranschreitende Internationalisierung bzw. Europäisierung des Rechts für die Regulierung von Strahlen prägend war und einen Harmonisierungsdruck ausübte, dem sich die Schweiz weder entziehen konnte noch wollte. Den notwendigen politischen Anstoß für eine stärkere Strahlenregulierung, insbesondere im Bereich der Radioaktivität von Lebensmitteln, hatte indessen das Ereignis von Tschernobyl und die damit verbundene mediale und öffentliche Aufmerksamkeit gegeben.

\section{Alarmieren}

Bereits vor dem Reaktorunfall in Tschernobyl bestanden Reformabsichten bezüglich des nuklearen Alarmierungsdispositivs. Mit dem Bau der Anlage "Metalert" war im Herbst 1984 die Nationale Alarmzentrale bei der Schweizerischen Meteorologischen Zentralanstalt in Zürich verwirklicht worden. Im Normalfall führte die bereits 1981 geschaffene Sektion Überwachungszentrale des EDI deren Betrieb; im Notfall konnte diese durch einen Armeestabsteil verstärkt werden. Für die fachliche Leitung der Nationalen Alarmzentrale war die Eidgenössische Kommission für AC-Schutz (KAC)

45 Ebd.

46 CH-BAR\#E $3310 A \# 2003 / 209 \# 44^{*}$, Antrag des EDI, 2.6.1994.

47 Vgl. Tanner 2015, S. 507-511. 
zuständig, welche den Alarmausschuss für den Fall erhöhter Radioaktivität sowie den Ausschuss AC-Schutz des Stabes für Gesamtverteidigung ersetzte. ${ }^{48}$

Um diese technischen Verbesserungen und strukturellen Neuerungen der Alarmorganisation rechtlich festzuhalten, sollte die Verordnung über die Alarmorganisation, die noch aus dem Jahr 1966 stammte, angepasst werden. Als sich die Nuklearkatastrophe von Tschernobyl ereignete, stand die Änderung dieser Verordnung kurz vor dem Abschluss. Aufgrund der Erfahrungen mit dem Reaktorunfall von Tschernobyl wurde dieses Revisionsvorhaben im Rahmen des 12-Punkte-Programmes des Bundesrates nochmals überarbeitet, was in einen neuen Verordnungsentwurf mündete, der Ende November 1986 vorlag. ${ }^{49}$ Die anvisierte Neustrukturierung der Alarmorganisation erwies sich indessen als stark problembehaftet, sodass hinsichtlich der Vorbereitung für einen atomaren Notfall Ende der 198oer Jahre eine regelrechte Revisionskaskade in Gang kam.

Die Verordnung über die Einsatzorganisation bei erhöhter Radioaktivität, die am 1. Mai 1987 in Kraft trat, ${ }^{50}$ nahm zwei wesentliche organisatorische Neuerungen auf, mit denen der Bund derjenigen Kritik Rechnung tragen wollte, welche Politik, Medien und Kantone im Zusammenhang mit der Bewältigung des Ereignisses von Tschernobyl vorgebracht hatten: Erstens oblag die Leitung der Alarmorganisation - nun Einsatzorganisation genannt für atomare Notfälle nicht mehr der KAC, sondern der Bundesverwaltung. Dadurch sollte - so hieß es in den Erläuterungen zum Verordnungsentwurf ein "nahtloser Übergang sowohl vom Normalzustand ins Krisen-Management als auch umgekehrt" gewährleistet werden. ${ }^{51}$ Zweitens war die Information der Medien und der Bevölkerung nun in allen Fällen Sache einer Informationszentrale der Bundeskanzlei, welche auch für den Informationsfluss zu den Kantonsregierungen und die Nachrichtenbeschaffung verantwortlich zeichnen sollte. ${ }^{52}$

Die neue Einsatzorganisation bestand aus drei Teilen: Die Gesamtleitung der erste Teil - lag in den Händen des Leitenden Ausschusses Radioaktivität. Diesem ämterübergreifenden Ausschuss gehörten unter der Federführung des EDI die Direktoren aller Bundesämter an, die von einem atomaren Ereignis

48 Vgl. dazu Kapitel 6.2.

49 Vgl. CH-BAR\#E3310A\#2003/209\# $47^{*}$, Schreiben von Ed. Marthaler an die mitinteressierten Bundesstellen, 25.11.1986, und Ämterkonsultation zum Entwurf der Verordnung zum Schutz der Bevölkerung bei erhöhter Radioaktivität, ohne Datum. Vgl. CH-BAR\#E3310A\#2003/209\#49*, Verordnung über die Einsatzorganisation bei erhöhter Radioaktivität, 15.4.1987.

51 CH-BAR\#E3310A\#2003/209\#47*, Ämterkonsultation zum Entwurf der Verordnung zum Schutz der Bevölkerung bei erhöhter Radiktivität, ohne Datum. 
in erster Linie betroffen wären, so unter anderem das BAG, das Bundesamt für Energiewirtschaft und das Bundesamt für Zivilschutz. ${ }^{53}$ Über die entsprechenden Departemente sollte auch eine direkte Verbindung zum Bundesrat gewährleistet sein. Aufgabe des Leitenden Ausschusses Radioaktivität war es, im Notfall die Gesamtlage zu beurteilen, beim Bundesrat Anträge für Schutzmaßnahmen zu stellen und den Vollzug der angeordneten Maßnahmen zu überwachen. ${ }^{54}$

Der Leitende Ausschuss Radioaktivität wurde vom Stab Gesundheitsschutz bei erhöhter Radioaktivität - dem zweiten Teil der neuen Einsatzorganisation - unterstützt. Das EDI erläuterte dazu, das Ereignis von Tschernobyl habe gezeigt, dass die KAC zwar „von richtigen theoretischen Voraussetzungen ausgegangen ist, in der Praxis aber überfordert war, weil sie vor allem personell über eine zu schmale Basis verfügte und deshalb auch nicht alle wesentlichen und bei A-Ereignissen relevanten Fachrichtungen ab-

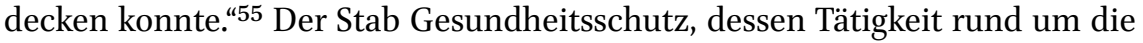
Uhr gewährleistet sein musste, und der, um die permanente Einsatzbereitschaft zu erleichtern, als Armeestabsteil organisiert war, sollte diese Lücke nun füllen. ${ }^{56}$ Als Chef des Stabes Gesundheitsschutz fungierte der Direktor des BAG; als Mitglieder amteten je zwei Vertreter der KAC, der KUeR, der EKS und der Eidgenössischen Kommission für die Sicherheit der Atomanlagen sowie der Abteilung Strahlenschutz und der Hauptabteilung für die Sicherheit der Kernanlagen. ${ }^{57}$ Der Stab Gesundheitsschutz, in welchen somit alle wesentlichen in dieser Arbeit behandelten Strahlenschutzbehörden und -gremien eingebunden waren, sollte im Notfall die radiologische Lage im Hinblick auf die Gesundheit der Bevölkerung beurteilen und für den Leitenden Ausschuss Radioaktivität Anträge für entsprechende Schutzmaßnahmen ausarbeiten. Der Stab war somit als fachliches Führungsorgan der neuen Einsatzorganisation vorgesehen. ${ }^{58}$

Den dritten Teil der neuen Einsatzorganisation stellten die „weiteren Mittel“ dar. Dazu zählte in erster Linie die Nationale Alarmzentrale. In diese war die

53 Vgl. CH-BAR\#E331OA\#2003/209\#48*, Pressemitteilung betreffend Verordnung über Einsatzorganisation bei erhöhter Radioaktivität, ohne Datum.

54 Vgl. ebd., Antrag des EDI, 8.4.1987.

55 Ebd.

56 Vgl. ebd.

57 Vgl. CH-BAR\#E3310A\#2003/209\#49*, Verordnung über die Einsatzorganisation bei erhöhter Radioaktivität, 15.4.1987. Die Vertreter der vier mit Radioaktivität und Strahlenschutz befassten Kommissionen bildeten darüber hinaus schon in der Vorbereitungsphase, also im Normalfall, den sogenannten Koordinationsausschuss Radioaktivität.

Vgl. CH-BAR\#E3310A\#2003/209\#48*, Antrag des EDI, 8.4.1987. 
schon bestehende Alarmstelle Radioaktivität integriert, die rund um die Uhr Meldungen aus dem In- und Ausland entgegenzunehmen und bei einem Ereignis unverzüglich den Pikettdienst der Sektion Überwachungszentrale zu informieren hatte. Bei Bedarf konnte zur Verstärkung zusätzlich der Armeestabsteil der Nationalen Alarmzentrale aufgeboten werden. ${ }^{59} \mathrm{Zu}$ den „weiteren Mitteln" der Einsatzorganisation gehörten nach wie vor auch die Messorganisation sowie die bereits erwähnte, bei der Bundeskanzlei angesiedelte neue Informationszentrale, die ebenfalls als Armeestabsteil organisiert war, sowie ein Radio-Detachement der Schweizerischen Rundspruchgesellschaft, das sich gleichzeitig mit dem Armeestabsteil der Nationalen Alarmzentrale aufbieten ließ. ${ }^{60}$ Wiewohl bestimmte Reformabsichten schon im Raum gestanden hatten, führte der Reaktorunfall von Tschernobyl also auch bei der Alarmorganisation zu einem Reorganisationsdruck und entsprechenden Umstrukturierungen.

Die vorgenommenen strukturellen Änderungen betrafen vor allem die Leitungsebene der neuen Einsatzorganisation. Das EDI hielt dazu fest: „Mit einer Kombination von Verwaltungseinheiten und ,Milizpersonal' wird ein genügend grosses Potential von Fachwissen zur Bewältigung einer A-Krise geschaffen, ohne einen bürokratischen Apparat unterhalten zu müssen.“61 Es steht jedoch außer Frage, dass die neue Einsatzorganisation auf eine Professionalisierung und damit auf eine Zurückdrängung der Milizorgane abzielte, sollten die Entscheidungen doch nicht mehr durch eine Kommission, sondern durch Verwaltungsorgane getroffen werden. Der KAC kam, wie den anderen Kommissionen im Bereich Radioaktivität und Strahlenschutz auch, nur noch eine beratende, jedoch keine Führungsfunktion mehr zu. In einem Zeitungsartikel der Luzerner Neuen Nachrichten von Ende April 1987 hieß es lapidar, gemäß der neuen Verordnung werde "die vor einem Jahr federführende und stark kritisierte Kommission für AC-Schutz weitgehend entmachtet [...]“. ${ }^{62}$ Hier zeigt sich somit erneut der Bedeutungsverlust außerparlamentarischer Expertenkommissionen.

Gleichzeitig verdeutlicht die neue Einsatzorganisation, wie stark das Bedrohungsbild einer Atomkatastrophe, die sich zu Friedenszeiten ereignen würde, inzwischen das Denken der Verantwortlichen bestimmte. Die Führung des Leitenden Ausschusses Radioaktivität lag nun beim EDI und diejenige

59 Am 1.1.1989 wurde die Sektion Überwachungszentrale in Sektion Nationale Alarmzentrale umbenannt. Vgl. E330oC\#1996/294\#842*, Schreiben von H. J. Knaus an Ed. Marthaler, 24.11.1988.

6o Vgl. CH-BAR\#E3310A\#2003/209\#48*, Antrag des EDI, 8.4.1987.

61 Ebd.

62 Ebd., Radioaktivität und Krisenfall, in: Luzerner Neue Nachrichten, 27.4.1987. 
des Stabes Gesundheitsschutz beim Direktor des BAG, also bei zwei zivilen Organen. Bemerkenswert ist auch, dass mit dem Begriff Gesundheitsschutz eine Bezeichnung gewählt wurde, die keinerlei militärische Konnotationen mehr aufwies. Dementsprechend standen jetzt eindeutig zivile Nuklearkatastrophen wie Kernkraftwerkunfälle oder Unfälle mit Atomwaffen im Fokus atomarer Bedrohungsvorstellungen. ${ }^{63}$ Auf diese atomaren Gefahren sollte das neue Alarmierungsdispositiv der Einsatzorganisation nun reagieren. Das Bedrohungsszenario eines Nuklearkrieges trat demgegenüber - beschleunigt durch das sich abzeichnende Ende des Kalten Krieges - erneut stark in den Hintergrund.

Die Reform der ehemaligen Alarmorganisation erwies sich jedoch als vertrackte Angelegenheit. Wie die obige Beschreibung klar gemacht hat, wies die nach Tschernobyl neu geschaffene Einsatzorganisation eine äußerst komplizierte, wenn nicht gar unübersichtliche Struktur auf. Diese wurde anlässlich der Gesamtverteidigungsübung des Jahres 1988, der letzten während des Kalten Krieges, getestet. Aufgrund der Erfahrungen mit dem Reaktorunfall von Tschernobyl sah die Übung eine "Mehrfachkrise“ vor. Bei deren Bewältigung unterstützte die Armee die zivilen Schutz- und Rettungsdienste und nahm insofern die spätere Neuausrichtung der sogenannten Armee 95 vorweg. ${ }^{64}$ Allerdings zeigten sich im Bereich der atomaren Bedrohung - wie in sämtlichen nationalen Verteidigungsübungen während des Kalten Krieges wiederum zahlreiche Koordinationsprobleme. So funktionierten zwar die einzelnen Elemente der neuen Einsatzorganisation für sich allein gut, doch es gab Schwierigkeiten in deren Zusammenspiel. Dies hatte in der Übung, in welcher laut der Übungsanlage ein Nuklearereignis im benachbarten Ausland stattfand, eine späte Alarmierung der Bevölkerung sowie fehlende oder unklare Anweisungen zur Folge. Eine Überprüfung der neuen Organisation schien deshalb angebracht. ${ }^{65}$ Tatsächlich wurde die Verordnung über die Einsatzorganisation bei erhöhter Radioaktivität bereits ab Mitte 1989 einer erneuten Revision unterzogen.

Es wird hier somit einmal mehr deutlich, welch zentrale Rolle Übungen oder genauer formuliert: Simulationen - für die Weiterentwicklung von Alarmorganisationen und Strahlenschutzmaßnahmen zukam. Im Simulationsprozess gewonnene Resultate und Erkenntnisse wurden häufig in

63 Vgl. CH-BAR\#E3310A\#2003/209\#47*, Schreiben von Mitgliedern der Ei Gr KAC und der NAZ an A. Egli, 3.9.1986, und Der Einsatz der Alarmorganisation beim Ereignis Tschernobyl, [20.10.1986].

64 Vgl. Bericht des Bundesrat 1992.

65 Vgl. CH-BAR\#E568oC\#1999/267\#73*, Feststellungen des zivilen Übungsleiters, 20.11.1988. 
konkrete Vorhaben übersetzt. Dies geschah auch nach der Gesamtverteidigungsübung 1988. Diese und weitere Übungen hätten - so ist den Erläuterungen zum neuen Verordnungsentwurf zu entnehmen - „die Bedeutung des Faktors ,Zeit‘ mit aller Deutlichkeit in den Vordergrund gerückt“. Es gelte nun deshalb,

die an sich reibungslos funktionierende Organisation zu straffen, was auf dem Wege der Eliminierung von Schnittstellen zu erfolgen habe. Insbesondere bei einem Unfall in einem schweizerischen Kernkraftwerk sei die Interventionsgrenze zu Beginn eines Ereignisses so zu ziehen, dass in längestens zwei Stunden erste entscheidende Schutzmassnahmen angeordnet werden können. ${ }^{66}$

Es waren also die vielen "Schnittstellen“ in der komplexen Struktur der Einsatzorganisation, die nicht richtig zusammenspielten und deren Abstimmung und Einbindung zu viel Zeit kosteten. Eine „Eliminierung“ bedeutete nichts anderes, als dass der Stab Gesundheitsschutz, kaum eingeführt, bereits wieder abgeschafft wurde. In der revidierten Verordnung über die Einsatzorganisation bei erhöhter Radioaktivität, die ab Juli 1991 in Kraft war, bestand die Einsatzorganisation nur noch aus zwei Teilen: der Nationalen Alarmzentrale und dem Leitenden Ausschuss Radioaktivität. Letzterer sollte die notwendigen fachlichen Einschätzungen zur radiologischen Situation künftig direkt bei den entsprechenden Bundesämtern abholen. ${ }^{67}$

Die bestehenden Milizkommissionen auf dem Gebiet der Radioaktivität und des Strahlenschutzes, insbesondere die KAC, wurden zu Beginn der 199oer Jahre folglich weiter „entmachtet“. Sie waren seit 1987 sowieso nur noch im Stab Gesundheitsschutz vertreten gewesen - und dieser fiel nun weg. Gemäß der neuen Verordnung standen die Mitglieder der vier Expertenkommissionen dem Leitenden Ausschuss Radioaktivität lediglich noch als Fachleute zur Verfügung. ${ }^{68}$ Zugleich spiegelt die bereits Mitte der 1980er Jahre einsetzende Revisionskaskade bei der Verordnung der Einsatzorganisation die anhaltenden Koordinationsprobleme im Bereich der Alarmierung und Information der Bevölkerung bei Atomkatastrophen wider. Augenscheinlich bildete die ,koordinierte Sicherheit' gegen die von Strahlen ausgehenden Gefahren während des gesamten Kalten Krieges (und darüber hinaus) ein unlösbares Problem.

66 CH-BAR\#E330OC\#2002/4O\#561*, Erläuterungen zum Entwurf der Verordnung über die Einsatzorganisation bei erhöhter Radioaktivität, 19./21.6.199o.

67 Vgl. ebd.

68 Vgl. ebd. 


\subsection{Fazit}

Der Reaktorunfall von Tschernobyl gab den Ausschlag dafür, die Sicherheitsdispositive für das Regieren von Strahlen im Normalfall wie im Notfall maßgeblich zu reformieren. Die Aufwertung der Sektion für Strahlenschutz zu einer Abteilung, die Schaffung der Sektion Überwachung der Radioaktivität, die Übernahme der Lebensmittelüberwachung durch den Verband der Kantonschemiker, der Erlass eines separaten Strahlenschutzgesetzes und einer neuen Strahlenschutzverordnung sowie die neu geschaffene Einsatzorganisation bei erhöhter Radioaktivität verdeutlichen die Aufwertung des Strahlenschutzes in der Schweiz am Ende der 1980er bzw. Anfang der 199oer Jahre. All diese Entwicklungen wurden durch den Reaktorunfall von Tschernobyl entweder entscheidend befeuert oder sogar erst angestoßen. Tschernobyl fungierte für das Regieren von Strahlen in der Schweiz somit zwar nicht als eine Zäsur, aber als ein äußerst wichtiger Treiber und Verstärker von Reformbestrebungen.

Sowohl die Organisation im Normalfall als auch die Vorbereitung auf einen atomaren Notfall wurden dabei professionalisiert. Was die Herstellung von Strahlensicherheit anbelangte, offenbarten die Auswirkungen des Reaktorunfalls von Tschernobyl auf die Schweiz die Schwächen von Milizkommissionen. Als Folge verlor das schweizerische Strahlenschutznetzwerk mit seinen milizbasierten Expertenkommissionen zugunsten professioneller Verwaltungseinheiten an Bedeutung und Einfluss. Die Strahlenüberwachung und die Alarmorganisation wurden reorganisiert und in die Verwaltung eingegliedert. Dies war in den 1980er Jahren auch deshalb möglich, weil die Verwaltung nun im Gegensatz zum Beginn des Kalten Krieges selbst über Know-how und Strahlenwissen verfügte. Die ,Koordination“ von Sicherheit blieb indessen, wie die Gesamtverteidigungsübung von 1988 zeigte, weiterhin ein struktureller Schwachpunkt des schweizerischen Gesamtverteidigungssystems, welcher bereits nach kürzester Zeit zu wiederholten Reformen der neuen Einsatzorganisation Anlass gab.

Darüber hinaus führte der Reaktorunfall von Tschernobyl dazu, dass der Bau des Kernkraftwerkes Kaiseraugst, das Pièce de Résistance der AntiAtomkraft-Bewegung, aufgegeben wurde. Auch in befürwortenden Kreisen büßte die Förderung der Atomenergie ihre vormalige uneingeschränkte Unterstützung ein. Damit erodierte als direkte Folge des Ereignisses von Tschernobyl ein zentraler Pfeiler des schweizerischen Kalte-Krieg-Konsenses. Strahlenhistorisch gesehen endete der schweizerische Kalte Krieg im Jahr 1986. 